\title{
NÍVEL DE ADERÊNCIA AOS INDICADORES AMBIENTAIS (GRI) DE UMA EMPRESA DE FRUTICULTURA DO VALE DO SÃO FRANCISCO
}

\author{
Marcos Victor do Carmo Loiola ${ }^{1}$ \\ Alvany Maria dos Santos Santiago ${ }^{2}$
}

\begin{abstract}
RESUMO
As empresas precisam adotar uma postura proativa e inovadora, frente às mudanças de um cenário globalizado, que permita melhor engajamento econômico-social e ambientalmente responsável. Indicadores com informações transparentes podem nortear o interesse e as decisões estratégicas dos diversos setores. O objetivo deste trabalho foi gerar o Grau de Aderência Plena (GAPIE) e o Grau de Evidenciação Efetiva (GEE) aos indicadores ambientais, baseados no Global Reporting Initiative (GRI) versão G4 de uma empresa de Fruticultura Irrigada do Vale do São Francisco em Petrolina-PE. A pesquisa teve caráter qualitativo e alcance exploratório-descritivo, sendo um estudo de caso. Os relatórios públicos de certificação ambiental, entre 2016 - 2018, foram obtidos na página institucional da empresa. Os dados foram analisados e tabulados por meio de planilha Excel com inserção de fórmulas automáticas desenvolvidas para este estudo. Foram analisados 34 indicadores. A empresa apresentou $61 \%$ de aderência, nível "B", e classificação "Médio", considerada como um bom grau de aderência aos indicadores ambientais. A divulgação dos indicadores de sustentabilidade das empresas do Vale do São Francisco, no Semiárido nordestino, é de peculiar importância a fim de que se tenha uma gestão mais efetiva pelas empresas e para que as políticas públicas caminhem em parceria com o setor empresarial e anseios da sociedade no quesito da sustentabilidade.
\end{abstract}

Palavras-chaves: Gestão ambiental; Relatório de sustentabilidade; Semiárido.

1 Doutorando do Programa de Pós-Graduação em Agroecologia e Desenvolvimento Territorial (PPGADT), em nível Doutorado Profissional, que se constitui numa associação de Universidades da Região Nordeste do Brasil, formada pela UNIVASF, UFRP, Universidade do Estado da Bahia UNEB. Contato: mloiola@gmail.com. Mestre em Dinâmicas de Desenvolvimento do Semiárido (PPGDiDeS/UNIVASF). Pesquisador selecionado no Edital n 01/2021 DE Bolsista da Fundação de Amparo à Pesquisa do Estado da Bahia (FAPESB).

${ }^{2}$ Professor of Management \& Human Resources, Ethics and Corporate Sustainability Responsibility and Sustainable Development Graduate Program in Dynamics of Development in Brazil Semi-arid region. http://www.ppgdides.univasf.edu.br/site/index.php/en/ Graduate Program in Public Management (Profiap Univasf) Federal University of Sao Francisco Valley (Univasf).

Leader of the research group Laboratory of Career and Competences Development (LCDC) and member of the researcher group Governance for Sustainability and Carbon Market Management (GpS) at Federal University of Bahia http://grupogagmc.blogspot.pt/p/blog-page_27.html http://orcid.org/0000-0002-5869-4848 E-mails: alvany.santiago@univasf.edu.br. 


\section{ADHERENCE TO ENVIRONMENTAL INDICATORS (GRI) OF A FRUITULTURE COMPANY IN THE SAN FRANCISCO VALLEY ABSTRACT}

Companies need to take a proactive and innovative stance in the face of changes in a globalized scenario that enables better economic, social and environmentally responsible engagement. The objective of this work was to generate the Full Adherence Degree (GAPIE) and the Effective Disclosure Degree (GEE) to the environmental indicators, based on the Global Reporting Initiative (GRI) (2013), of Irrigated Fruit Company of the São Francisco Valley, in Petrolina-PE. The research had qualitative character and exploratory-descriptive scope, and is a case study. The public environmental certification reports, from 2016 - 2018, were obtained from the company's institutional page. Data were analyzed and tabulated using Excel spreadsheet with automatic formulas developed for this study. Thirty-four indicators were analyzed. The company presented $61 \%$ adherence, level "B", and rating "Medium", considered as a good degree of adherence to environmental indicators. The disclosure of sustainability indicators for companies in the São Francisco Valley, Northeastern Semiarid, is of particular importance in order to have a more effective management by companies and for public policies to go hand in hand with the business sector and society's demands for sustainability.

Key-words: Environmental management; Sustainability report; Semiarid.

\section{INTRODUÇÃO}

A modernização, a globalização, o avanço tecnológico, o crescimento econômico e o rápido acesso às informações têm causado grandes impactos na sociedade. Concomitantemente, temas como a degradação dos recursos naturais, da biodiversidade, mudanças climáticas vêm destacando a importância do envolvimento da sociedade civil e empresarial na questão ambiental e sustentabilidade.

As empresas devem adotar uma postura proativa e inovadora, frente às mudanças de um cenário globalizado, que permita melhor engajamento econômicosocial e ambientalmente estratégico e responsável (TRAVASSOS et al., 2014).

Sendo assim, a empresa, ao preocupar-se com o meio ambiente, deve utilizar métricas, indicadores ambientais que estejam relacionados à gestão ambiental e que sejam coerentes com o ecossistema. O relatório da Global Reporting Initiative (GRI), no qual esta pesquisa se fundamenta, é constituído por princípios e indicadores de ordem ambiental, com ênfase na transparência, qualidade e confiabilidade das informações divulgadas (GRI, 2006). 
A GRI configura-se como uma iniciativa conjunta entre a Coalizão por Economias Ambientalmente Responsáveis - Coalition for Environmentally Responsible Initiatives (CERES) e o Programa Ambiental das Nações Unidas - United Nations Environment Program (UNEP), em 1997, com o intuito de aumentar a qualidade, o rigor e a utilidade de relatórios para a sustentabilidade corporativa (STROBEL, 2005). Foi criado em 2020, um conselho consultivo da GRI no Brasil que se dedicará aos padrões para relatórios de sustentabilidade e vai montar um ranking de empresas mais transparentes prometido até o fim do corrente ano (TAUHATA, 2020).

O Instituto Brasileiro de Relações com Investidores (IBRI), em 2012, expôs que $62 \%$ das empresas emitem relatório de sustentabilidade, 17\% pretendem emiti-lo e $21 \%$ não o fazem. Observa-se também que a maioria das empresas (79\%) utiliza o padrão internacional GRI na hora de relatar suas ações socioambientais para empresários e consumidores (IBRI, 2012).

Dados recentes do Instituto Brasileiro de Geografia e Estatística (IBGE) que acompanhou 116,9 mil empresas, pela Pesquisa de Inovação (Pintec), observou que dessas apenas 4,8 mil publicaram relatórios de sustentabilidade no período de 2015 a 2017 , correspondendo a $4,1 \%$ do total. O setor de eletricidade e gás apresentou os melhores números, pois havia 304 empresas do segmento com publicação do relatório, correspondendo a pouco mais da metade $(51,2 \%)$ das companhias acompanhadas (IBGE, 2020).

Considerando as informações sobre os aspectos econômicos, ambientais e sociais das atividades empresariais, esta pesquisa centrou-se na dimensão ambiental, frente aos indicadores de matéria-prima, energia, água, geração de resíduos, emissões atmosféricas, efluentes líquidos e infraestrutura. A categoria ambiental também auxilia em melhorias na garantia de obtenção e elevação no montante da renda auferida, na dedicação e no perfil do responsável pelo estabelecimento e no relacionamento institucional.

Assim, o objetivo deste trabalho é verificar o grau de aderência aos indicadores ambientais, padrão GRI, de uma empresa do setor da fruticultura irrigada no Vale do São Francisco. 


\section{REFERENCIAL TEÓRICO}

\subsection{GESTÃO AMBIENTAL}

As empresas são cobradas, cada vez mais, pelos stakeholders por um comportamento socioambiental responsável, para evitar que os seus processos produtivos para mitigar as problemáticas já instaladas e, mesmo, evitar um colapso do ecossistema. Tal responsabilidade encontra-se no âmago da gestão empresarial, tendo caráter estratégico, não só em função da crescente ampliação da consciência ecológica da sociedade, mas também pela necessidade de as empresas se manterem competitivas no mercado globalizado.

A Gestão Ambiental pode ser definida como a forma de gerir, administrar, dirigir ou conduzir os ecossistemas naturais e sociais, incluindo o ser humano que compõe o meio de vida, em uma dinâmica de interação entre as atividades exercidas, buscando a conservação dos recursos naturais e das características do entorno com o propósito de manter o equilíbrio entre natureza e ser humano (PHILIPPI; BRUNA, 2004).

A Gestão Ambiental está inserida nas agendas pública e privada, ao redor do mundo, sendo reconhecida como fator fundamental para o sucesso das organizações. O desempenho ambiental de uma empresa é resultado da ação de três áreas da organização: administrativa, operacional tecnológica e a ambiental. A interação entre elas e a consolidação dessas informações seguindo os indicadores, podem permitir uma avaliação integral do desempenho ambiental da organização (CNTL, 2001).

De acordo com Nunes-Maia (2000), para uma gestão ambiental eficiente, é imprescindível considerar as variáveis econômicas, sociais, culturais e ambientais, para que possam alimentar um planejamento estratégico consistente. Essas perpassam pela formulação da estratégia em meio ambiente, pela definição das informações, controles e indicadores ambientais de desempenho a serem adotados, até o acompanhamento e a avaliação dos resultados obtidos.

Internacionalmente, a gestão ambiental repercute como resposta a uma crise ambiental sistêmica de grandes proporções, que atinge todos os países, 
principalmente países industrializados. A sociedade que contribui para a destruição do planeta também deve se responsabilizar pela gestão ambiental.

Ribas et al., (2017) também corroboram que devemos utilizar meios para desenvolver atitudes que sejam contrárias à devastação dos recursos naturais presentes em nossos ecossistemas e ambiente naturais, afirmando que a utilização de combustíveis fósseis está entre as principais fontes geradoras de dióxido de carbono, bem como as queimadas de florestas e de resíduos. Todas essas ações são desencadeadas, direta ou indiretamente, por organizações que, atualmente, fazem parte do problema, sendo necessário que, urgentemente, passem a fazer parte da solução.

A gestão ambiental é uma prática que vem se desenvolvendo de forma considerável nas últimas décadas, como resultado da necessidade de adequação a essa nova forma de pensar em desenvolvimento e produção de bens de consumo, circunscrita pelo desenvolvimento sustentável. Assim, a gestão ambiental requer uma abordagem interdisciplinar, que integre as ciências exatas, biológicas e humanas ao planejamento e às políticas públicas.

\subsection{INDICADORES AMBIENTAIS}

O uso de indicadores ambientais auxilia a uma maior compreensão dos aspectos necessários para possibilitar a mitigação dos danos ambientais das organizações. Polaz e Teixeira (2009) afirmam que investir na implantação de um sistema de indicadores locais é uma forma de munir a sociedade com um instrumento que, dentre outras características, tem um aspecto educacional altamente relevante. Muito além de um mero instrumento de gestão, os indicadores podem ser interpretados como importantes ferramentas pedagógicas, uma vez que despertam a atenção e o interesse da sociedade para os fenômenos que são evidenciados.

No entanto, é indispensável que o indicador tenha validade, pois é necessário que apresente metas, e essas demonstrem se os resultados da empresa estão compatíveis com o planejado previamente, verificando onde é necessário melhorar. O controle dos indicadores se torna imprescindível para avaliar o funcionamento dos sistemas de gestão e para garantir o cumprimento da política da empresa que vise 


\section{¿unisul}

principalmente à melhoria contínua dos sistemas de gestão (ALMEIDA; NUNES, 2014).

É preciso que os indicadores sejam utilizados não somente para a avaliação comparativa da qualidade de vida e do ambiente entre as cidades nas regiões estudadas, mas que possam constituir em ferramentas auxiliares no processo de planejamento de cidades e microrregiões, ao indicar as áreas de melhor ou pior desempenho relativo, apontar tendências e chamar a atenção para pontos fracos (BRAGA et al., 2004).

O conjunto de indicadores ambientais servirá para propiciar um melhor aproveitamento dos recursos naturais e também para indicação de medidas preventivas de degradação ambiental e consequentes prejuízos econômicos para a sua reparação. A informação que liga fatores socioeconômicos e ambientais oferece uma sólida base empírica para construir indicadores de sustentabilidade e, dessa maneira, medir os impactos e avaliar a eficiência sob vários pontos de vista dos processos de desenvolvimento (NETO; KRÜGER; DZIEDZIC, 2009).

\subsection{RELATÓRIO DE SUSTENTABILIDADE PADRÃO GRI}

A Global Reporting Initiative teve seu marco inicial com o primeiro balanço social da história, publicado em 1972, na França, pela companhia Singer, abrindo caminho para que, em 1977, fosse aprovada a Lei 77.769 que tornava obrigatória a publicação de balanços sociais por empresas francesas com mais de 700 funcionários.

No Brasil, o pioneirismo foi da estatal Nitrofértil, localizada na Bahia, que lançou seu balanço social em 1984. Contudo, foi a partir da década de 1990 que a publicação de balanços e relatórios começou a crescer no país, principalmente, a partir de 1997, com a campanha lançada por Betinho através do Instituto Brasileiro de Análises Sociais e Econômicas (lbase), com o objetivo de convocar as empresas à responsabilidade social.

O lbase criou um modelo que incluía dados contábeis, indicadores sociais e ambientais; porém, era baseado apenas em dados quantitativos, sem exigências quanto ao aprofundamento das ações relatadas (OLIVEIRA, 2017). 


\section{১unisul}

A GRI é uma organização não governamental internacional, com sede hoje em Amsterdã, Holanda, e elabora diretrizes e indicadores para o Relatório de Sustentabilidade (RS) com a missão de divulgar um modelo de evidenciação dos três desempenhos da empresa: social, econômico e ambiental (SANTOS; GONÇALVES, 2015). Essa tem por objetivo equiparar relatórios sociais e de sustentabilidade aos relatórios financeiros, bem como construir uma base comum sobre a qual se possa instituir uma estrutura consistente para a divulgação de informações sobre as dimensões econômicas, ambientais e sociais (MUELLER, 2003).

O Relatório de Sustentabilidade é um instrumento gerencial estratégico fundamental para a organização, pois proporciona mudanças para a gestão rumo a uma economia global sustentável que harmoniza a rentabilidade de longo prazo com o comportamento ético, cuidado ambiental e justiça social (PEREIRA et al., 2015).

No Brasil, a Lei 13.303, de 30 de junho de 2016, em seu Art. 8º, inciso IX, prevê que as empresas públicas e as sociedades de economia mista deverão divulgar anualmente relatório integrado ou de sustentabilidade.

A partir de 2004, com o apoio do Instituto Ethos de Responsabilidade Social Empresarial, foi possível realizar a publicação do relatório da GRI em língua portuguesa. Destaca-se que a empresa Natura foi a primeira empresa brasileira a publicar relatórios de acordo com as diretrizes da GRI.

Há mais de 8000 empresas no mundo que adotam a GRI, incluindo 88 das 100 maiores empresas brasileiras: AmBev, Banco Itaú, CPFL Energia, Eco rodovias, Itaipu Binacional, McDonald's, Natura, Petrobras, Siemens, Unilever, Votorantim e Walmart, entre outras. O conjunto de princípios, protocolos e indicadores desenvolvido pela GRI torna possível gerir, comparar e comunicar o desempenho das organizações nas dimensões social, ambiental e econômica (SGS, 2019).

Rocha (2012) analisou os relatórios de sustentabilidade das empresas Duratex, Natura, Samarco e Unilever, referentes ao exercício de 2011, disponibilizados em seus sítios institucionais. Constatou-se que vários indicadores ambientais não foram identificados.

Carvalho e Siqueira (2007) ressaltam que a elaboração e a publicação de informações não são obrigatórias, mas é notavelmente crescente a utilização do 
balanço social pelas organizações. É preciso que tais informações permitam tecer comparações do desempenho atual em relação a períodos anteriores da própria empresa, de forma que fique clara sua evolução através dos tempos; bem como possibilite sua comparação em relação ao desempenho de outras empresas.

A pesquisa realizada por Dias (2006) observou o grau de aderência plena (GAPIE) aos indicadores essenciais estabelecidos pelo GRI nas empresas brasileiras. O GAPIE soma os indicadores com aderência plena (ADP) aos omitidos com justificativa (OJ).

Já Carvalho (2007), dando sequência ao trabalho de Dias (2006), analisou as empresas latino-americanas, e desenvolveu o método Grau de Evidenciação Efetiva (GEE).

O método de cálculo do GEE é bastante similar ao do GAPIE, diferenciando-se apenas pela exclusão dos indicadores omitidos com justificativa (OJ) da equação (Figura 1). Os resultados das pesquisas desses dois autores corroboram para o fato de que existem divergências na padronização do relatório de sustentabilidade das empresas associadas ao GRI, mesmo com a disponibilização de diretrizes para a elaboração do mesmo.

Os autores Fernandes, Siqueira e Gomes (2010) reconsideraram os indicadores essenciais do GRI utilizados nos trabalhos de Dias (2006) e Carvalho (2007), por meio da separação dos mesmos em unidades de informação. Verificaram que os indicadores que antes não eram aproveitados, pelo fato de não possuírem todos os requisitos atendidos, quando separados em unidades de informação, puderam ter uso para investigação, ou seja, indicando a ausência de objetividade dos indicadores essenciais GRI.

Silva, Maia e Leal (2017) analisaram o grau de evidenciação aos indicadores ambientais dos relatórios de sustentabilidade do ano de 2013 de empresas listadas na Bolsa Mercantil de Futuros (BMF) \& Bovespa. Os resultados do estudo apontaram para o fato de que em mercados emergentes, as organizações não investem, a curto prazo, em decisões gerenciais sustentáveis, e que não há total transparência nas informações relativas à dimensão ambiental. 
Sucena e Marinho (2019) avaliaram o nível da evidenciação ambiental praticada nos relatórios de sustentabilidade das indústrias cervejeiras brasileiras nos anos de 2011 a 2013. Eles destacam que a divulgação de informações ambientais nos Relatórios de Sustentabilidade do setor cervejeiro possui fragilidades, dentre elas a baixa divulgação de indicadores GRI com aderência plena, e falta de correlação financeira dos indicadores ambientais.

O benefício da adesão ao Relatório de Sustentabilidade tem sido gradativamente adotado por várias empresas, considerando que confere maior transparência e integridade sobre o desempenho de sustentabilidade do seu segmento. A divulgação dessas informações torna-se uma importante força motriz para o desenvolvimento de uma relação melhor entre sociedade e meio ambiente.

\section{METODOLOGIA}

A pesquisa tem caráter documental, qualitativo e alcance exploratóriodescritivo, adotando, como estratégia de investigação, o estudo de caso único (YIN, 2001).

Como forma de olhar a realidade com seus limites e contextos, é que se chega a necessidade de escolher o estudo de caso como método de pesquisa. Segundo Yin (2001): "o estudo de caso é uma investigação empírica de um fenômeno contemporâneo dentro de um contexto da vida real, sendo que os limites entre o fenômeno e o contexto não estão claramente definidos". O autor enfatiza ainda, que esta é a estratégia mais escolhida quando é preciso responder a questões do tipo "como" e "por quê" e quando o pesquisador possui pouco controle sobre os eventos pesquisados.

Esse método investigativo é capaz de reunir, informações numerosas e detalhadas para aprender a totalidade de uma situação. Nesse sentido, os estudos de caso não buscam a generalização de seus resultados, mas sim a compreensão e interpretação mais profunda dos fatos e fenômenos específicos. Embora não possam ser generalizados, os resultados obtidos devem possibilitar a disseminação do conhecimento, por meio de possíveis generalizações ou proposições teóricas que possam surgir do estudo (YIN, 2001). O critério utilizado para a análise dos dados 
nesta pesquisa foi avaliar os relatórios de sustentabilidade elaborados nos anos de 2016 - 2018, disponíveis no site institucional de uma empresa de Fruticultura Irrigada do Vale do São Francisco em Petrolina - PE, classificados como fonte documental secundária.

O método de abordagem utilizado foi o dedutivo que, conforme expõe Andrade (2009), é composto por observações sequenciadas, que norteiam um raciocínio e direcionam a uma conclusão, a partir de teorias gerais.

As categorias utilizadas foram baseadas no GRI, versão 4.0, de 2013, e incluem os seguintes temas: materiais, energia, água, biodiversidade, emissões, efluentes e resíduos, produtos e serviços, conformidade, transportes, geral (total de novos investimentos e gastos com proteção ambiental), avaliação ambiental de fornecedores, mecanismos de queixas e reclamações relacionadas a impactos ambientais. Os aspectos e indicadores relacionados às questões sociais e econômicas não foram considerados neste estudo, já que se centrou na Categoria Ambiental apenas.

Foi realizada pesquisa bibliográfica, coleta de dados secundários em Rainforest Alliance CertifiedTM, Relatório de Auditoria para Administradores de Grupo Resumo Público digitais, análise das Certificações Ambientais e relatórios dos modelos de certificações nacionais, mundiais e de sustentabilidade. Acessando o site da empresa em estudo, foram obtidos os relatórios de sustentabilidade, nos quais se verificou a existência da divulgação do índice remissivo dos indicadores do GRI, segundo os padrões, que se subdividem em dois tipos: conteúdos padrão gerais e específicos. Os conteúdos gerais compreendem: a estratégia e análise, perfil organizacional, aspectos materiais identificados e limites, engajamento de Stakeholders, perfil do Relatório, governança, ética e integridade, e demanda a sua aplicabilidade a todas as organizações que redigem relatórios de sustentabilidade. Já os conteúdos específicos tratam da Forma de Gestão e dos Indicadores, e se apresentam em três categorias - econômica, ambiental e social. 


\subsection{TRATAMENTO DOS DADOS}

A análise dos relatórios consiste na comparação entre as informações solicitadas pelo indicador essencial GRI. A classificação das informações foi baseada em Castro, Siqueira, Macedo (2009), apresentado no Quadro 1.

O indicador requerido foi classificado como "apresentado" ou "não apresentado". Dentro dessa classificação, há uma divisão em categorias; o indicador "apresentado" pode ser categorizado como: com "aderência plena", com "aderência parcial", "dúbio", ou "inconsistente"; e o indicador "não apresentado" pode ser subdividido em: "não disponível", "não aplicável", "omitido com justificativa", ou simplesmente "omitido".

\section{Quadro 1 - Base para Classificação das Informações}

\begin{tabular}{|c|l|}
\hline \multicolumn{2}{|c|}{ APRESENTADOS } \\
\hline APL & $\begin{array}{l}\text { ADERÊNCIA PLENA: Quando todos os dados requeridos no protocolo do indicador essencial da G4 } \\
\text { foram devidamente fornecidos pela organização. }\end{array}$ \\
\hline AP & $\begin{array}{l}\text { ADERÊNCIA PARCIAL: Quando apenas parte dos dados requeridos no protocolo do indicador } \\
\text { essencial da G4 foram apresentados pela organização. }\end{array}$ \\
\hline D & $\begin{array}{l}\text { DÚBIO: Quando as informações fornecidas não são suficientes para o usuário avaliar se a } \\
\text { aderência é plena ou parcial. }\end{array}$ \\
\hline I & $\begin{array}{l}\text { INCONSISTENTE: Quando as informações fornecidas pela organização diferem daquelas } \\
\text { requeridas no protocolo do indicador essencial da G4. }\end{array}$ \\
\hline ND & $\begin{array}{l}\text { NÃO DISPONÍVEL: Quando a organização reconhece que a informação requerida é pertinente às } \\
\text { suas atividades, porém esta ainda não tem condição de fornecê-la. Quando a organização } \\
\text { reconhece que a informação requerida é pertinente às suas atividades, porém esta ainda não tem } \\
\text { condição de fornecê-la. }\end{array}$ \\
\hline NA & $\begin{array}{l}\text { NÃO APLICÁVEL: Quando a organização reconhece que os dados requeridos pelo indicador não } \\
\text { são pertinentes às suas atividades ou ao setor em que ela atua. }\end{array}$ \\
\hline OJ & $\begin{array}{l}\text { OMITIDO COM JUSTIFICATIVA: Quando a organização omite a informação requerida pelo } \\
\text { protocolo do indicador essencial da G4, por sua decisão, porém apresentando uma justificativa para } \\
\text { tal omissão. }\end{array}$ \\
\hline O & \begin{tabular}{l} 
OMITIDO: Quando nada é comentado sobre o indicador, como se o mesmo não existisse. \\
\hline
\end{tabular}
\end{tabular}

Fonte: Castro, Siqueira, Macedo (2009).

Uma vez que os indicadores essenciais informados pela empresa foram classificados, seguiu-se calculando o grau de aderência plena aos indicadores essenciais da GRI (GAPIE-GRI) e grau de evidenciação efetiva (GEE) (Figura 2). Este cálculo permite que se perceba o percentual de aderência de cada empresa ao que foi solicitado pela GRI, podendo esse ser de 0\% a $100 \%$.

A Figura 1 apresenta os graus de aderência para os indicadores da GRI, onde o grau de aderência plena aos indicadores essenciais é representado por GAPIE-GRI, 


\section{¿unisul}

grau de evidenciação efetiva é o GEE, APL é a aderência plena, os omitidos com justificativa é apresentado por OJ, e o NA é não aplicável.

Figura 1 - Base para Classificação dos Indicadores

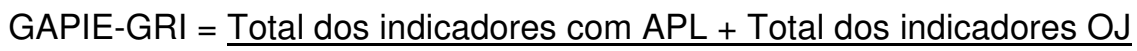 \\ Total dos indicadores essenciais - Total dos indicadores NA \\ GEE $=\underline{\text { Total dos indicadores com APL }}$}

Total dos indicadores essenciais - Total dos indicadores NA

Fonte: Castro, Siqueira, Macedo (2009)

Na Figura 1, no numerador, está o total dos indicadores omitidos com justificativa $(\mathrm{OJ})$ somado ao total de indicadores com aderência plena (APL) pelo fato de que a GRI permite a omissão de indicadores, desde que seja justificada. Note que quando OJ é zero, o GAPIE terá o mesmo valor que o GEE. Já no denominador, o total de indicadores não aplicáveis (NA) é subtraído do total de indicadores essenciais para não prejudicar o resultado do grau de aderência plena da empresa em função da existência de um indicador que não se aplica às suas atividades.

As diretrizes dos 34 indicadores da dimensão ambiental utilizada estão mostradas resumidamente nos Quadros 2 a 5.

Quadro 2 - Indicadores de materiais, energia e água do GRI.

G4 - DIRETRIZES PARA RELATO DE SUSTENTABILIDADE CATEGORIA: AMBIENTAL.

\begin{tabular}{|c|c|l|}
\hline \multirow{2}{*}{ Materiais } & G4-EN1 & Materiais usados, discriminados por peso ou volume \\
\cline { 2 - 3 } & G4- EN2 & Percentual de materiais usados provenientes de reciclagem \\
\hline \multirow{4}{*}{ Energia } & G4- EN3 & Consumo de energia dentro da organização \\
\cline { 2 - 3 } & G4- EN4 & Consumo de energia fora da organização \\
\cline { 2 - 3 } & G4- EN5 & Intensidade energética \\
\cline { 2 - 3 } & G4- EN6 & Redução do consumo de energia \\
\cline { 2 - 3 } & G4- EN7 & $\begin{array}{l}\text { Reduções nos requisitos de energia relacionados a produtos e } \\
\text { serviços }\end{array}$ \\
\hline \multirow{3}{*}{ Água } & G4- EN8 & Total de retirada de água por fonte \\
\cline { 2 - 3 } & G4- EN9 & Fontes hídricas significativamente afetadas por retirada de água \\
\cline { 2 - 3 } & G4- EN10 & Percentual e volume total de água reciclada e reutilizada \\
\hline
\end{tabular}


Quadro 3- Indicadores de biodiversidade, emissões e efluentes e resíduos do GRI

G4 - DIRETRIZES PARA RELATO DE SUSTENTABILIDADE CATEGORIA: AMBIENTAL.

\begin{tabular}{|c|c|c|}
\hline \multirow{4}{*}{ Biodiversidade } & G4- EN11 & $\begin{array}{l}\text { Unidades operacionais próprias, arrendadas ou administradas } \\
\text { dentro ou nas adjacências de áreas protegidas e áreas de alto } \\
\text { valor para a biodiversidade situadas fora de áreas protegidas }\end{array}$ \\
\hline & G4- EN12 & $\begin{array}{l}\text { Descrição de impactos significativos de atividades, produtos e } \\
\text { serviços sobre a biodiversidade em áreas protegidas e áreas de } \\
\text { alto valor para a biodiversidade situadas fora de áreas protegidas }\end{array}$ \\
\hline & G4- EN13 & Habitats protegidos ou restaurados \\
\hline & G4- EN14 & $\begin{array}{l}\text { Número total de espécies incluídas na lista vermelha da IUCN e } \\
\text { em listas nacionais de conservação com habitats situados em } \\
\text { áreas afetadas por operações da organização, discriminadas por } \\
\text { nível de risco de extinção }\end{array}$ \\
\hline \multirow{7}{*}{ Emissões } & G4- EN15 & Emissões diretas de Gases de Efeito Estufa (GEE) \\
\hline & G4- EN16 & $\begin{array}{l}\text { Emissões indiretas de Gases de Efeito Estufa (GEE) provenientes } \\
\text { da aquisição de energia }\end{array}$ \\
\hline & G4- EN17 & Outras emissões indiretas de Gases de Efeito Estufa (GEE) \\
\hline & G4- EN18 & Intensidade de emissões de Gases de Efeito Estufa (GEE) \\
\hline & G4- EN19 & Redução de emissões de Gases de Efeito Estufa (GEE) \\
\hline & G4- EN20 & $\begin{array}{l}\text { Emissões de substâncias que destroem a Camada De Ozônio } \\
\text { (SDO) }\end{array}$ \\
\hline & G4- EN21 & $\begin{array}{l}\begin{array}{l}\text { Emissões de NOX, SOX e outras emissões atmosféricas } \\
\text { significativas }\end{array} \\
\end{array}$ \\
\hline \multirow{5}{*}{$\begin{array}{l}\text { Efluente e } \\
\text { Resíduos }\end{array}$} & G4- EN22 & Descarte total de água, discriminado por qualidade e destinação \\
\hline & G4- EN23 & $\begin{array}{l}\text { Peso total de resíduos, discriminados por tipo e método de } \\
\text { disposição }\end{array}$ \\
\hline & G4- EN24 & Número total e volume de vazamentos significativos \\
\hline & G4- EN25 & $\begin{array}{l}\text { Peso de resíduos transportados, importados, exportados ou } \\
\text { tratados considerados perigosos nos termos da convenção da } \\
\text { brasileira, e percentual de resíduos transportados } \\
\text { internacionalmente }\end{array}$ \\
\hline & G4- EN26 & $\begin{array}{l}\text { Identificação, tamanho, status de proteção e valor da } \\
\text { biodiversidade de corpos d'água e habitats relacionados } \\
\text { significativamente afetados por descartes e drenagem de água } \\
\text { realizados pela organização }\end{array}$ \\
\hline
\end{tabular}

Fonte: Adaptado de GRI (2013).

Quadro 4 - Indicadores de produtos e serviços, conformidade e transporte e resíduos do GRI.

\begin{tabular}{|c|c|l|}
\hline G4 - DIRETRIZES PARA RELATO DE SUSTENTABILIDADE CATEGORIA: AMBIENTAL. \\
\hline $\begin{array}{c}\text { Produtos e } \\
\text { serviços }\end{array}$ & G4- EN27 & Extinção da mitigação de impactos ambientais de produtos e serviços \\
\cline { 2 - 3 } Conformidade & G4- EN29 & $\begin{array}{l}\text { Percentual de produtos e suas embalagens recuperados em relação } \\
\text { ao total de produtos vendidos, discriminados por categoria de } \\
\text { produtos }\end{array}$ \\
\hline $\begin{array}{l}\text { Valor monetário de multas significativas e número total de sanções } \\
\text { não monetárias aplicadas em decorrência da não conformidade com } \\
\text { leis e regulamentos ambientais }\end{array}$ \\
\hline Transporte & G4- EN30 & $\begin{array}{l}\text { Impactos ambientais significativos decorrentes do transporte de } \\
\text { produtos e outros bens e materiais usados nas operações da } \\
\text { organização, bem como do transporte de seus empregados }\end{array}$ \\
\hline
\end{tabular}

Fonte: Adaptado de GRI (2013).

Revista Gestão e Sustentabilidade Ambiental., v. 10, n. 3, p. 197-218, set. 2021. 


\section{GESTÃO \& SUSTENTABILIDADE AMBIENTAL

\section{sunisul}

Quadro 5 - Indicadores de geral, avaliação ambiental de fornecedores e mecanismos de queixas e reclamações relacionadas a impactos ambientais do GRI.

\begin{tabular}{|c|l|l|}
\hline \multicolumn{2}{|c|}{ G4 - DIRETRIZES PARA RELATO DE SUSTENTABILIDADE CATEGORIA: AMBIENTAL. } \\
\hline Geral & G4- EN31 & $\begin{array}{l}\text { Total de investimentos e gastos com proteção ambiental, discriminado } \\
\text { por tipo }\end{array}$ \\
\hline $\begin{array}{c}\text { Avaliação } \\
\text { ambiental de } \\
\text { fornecedores }\end{array}$ & G4- EN32 & $\begin{array}{l}\text { Percentual de novos fornecedores selecionados com base em } \\
\text { critérios ambientais }\end{array}$ \\
\cline { 2 - 4 } $\begin{array}{c}\text { Mecanismos de } \\
\text { queixas e } \\
\text { reclamações } \\
\text { relacionadas a } \\
\text { impactos } \\
\text { ambientais }\end{array}$ & G4- EN34 & $\begin{array}{l}\text { Impactos ambientais negativos significativos reais e potenciais na } \\
\text { cadeia de fornecedores e medidas tomadas a esse respeito }\end{array}$ \\
\hline
\end{tabular}

Fonte: Adaptado de GRI (2013).

Para a classificação dos resultados em alto, médio ou baixo, foram tomados, como base, os critérios referentes à apresentação dos indicadores requeridos para a classificação nos três níveis de aplicação sugeridos pela GRI ( $A, B$ e C), conforme apresentado na Figura 2.

Figura 2 - Níveis de Classificação do GAPIE e GEE

\begin{tabular}{|c|c|c|}
\hline Faixa & Nível & Classificação \\
\hline $0 \% \vdash 24 \%$ & "C" & Baixo \\
\hline $25 \% \vdash 61 \%$ & "B" & Médio \\
\hline $62 \% \vdash 100 \%$ & "A" & Alto \\
\hline
\end{tabular}

Fonte: Castro, Siqueira, Macedo (2009).

Dessa forma, para a empresa analisada ser classificada com alto grau, ou seja, como uma organização de grande porte, ela deve apresentar resultado acima de $61 \%$. Isso porque, para ser classificada como nível $\mathrm{A}$, deve ter respondido, pelo menos, os 22 indicadores essenciais de um total de 34 , o que equivale a uma proporção de aproximadamente $65 \%$.

Para uma empresa ser classificada como nível $\mathrm{B}$, deve responder a, pelo menos, 9 indicadores do total de 34 , o equivalente a aproximadamente $27 \%$, ou seja, a organização que apresenta um resultado entre $25 \%$ e $61 \%$ será classificada como 


\section{GESTÃO \& SUSTENTABILIDADE AMBIENTAL

\section{sunisul}

grau médio. O nível C (baixo) é considerado para empresas que exibirem um resultado inferior a 25\% (CASTRO; SIQUEIRA; MACEDO, 2009).

Os dados relativos a todos os indicadores foram inseridos e tabulados em planilha, Microsoft Excel, criada com as fórmulas GAPI e GEE especialmente para este estudo. Os resultados podem variar de $0 \%$, correspondente ao menor grau de aderência e evidenciação, e 100\%, que representa o maior grau.

\section{ANÁLISE E DISCUSSÃO DOS RESULTADOS}

Os resultados obtidos neste trabalho encontram-se apresentados no Quadro 7. Em que 1 classifica a Presença da Informação e 0 a ausência.

Dos 34 indicadores, 20 identificaram a Aderência Plena; 04 identificaram Aderência Parcial; 07 Não estavam disponíveis; 02 Não aplicáveis e 01 Dúbio.

Após a classificação das respostas dos indicadores essenciais, foi gerado 0 cálculo dos Graus de Aderência Plena (GAPIE) e Grau de Evidência Efetiva (GEE). As classificações nas categorias "não disponível" e "não aplicável" são frutos do relato da própria organização. O total dos indicadores Omitidos com Justificativa (OJ) foi zero, o que igualou o GAPIE e GEE, representados numa única coluna na Tabela 1.

Quadro 7 - Apresenta os Resultados dos Indicadores Ambientais do GRI. APLAderência Plena; AP-Aderência Parcial; ND-Não Disponível; NA-Não Aplicável; DDúbio. EN-Environmental Category

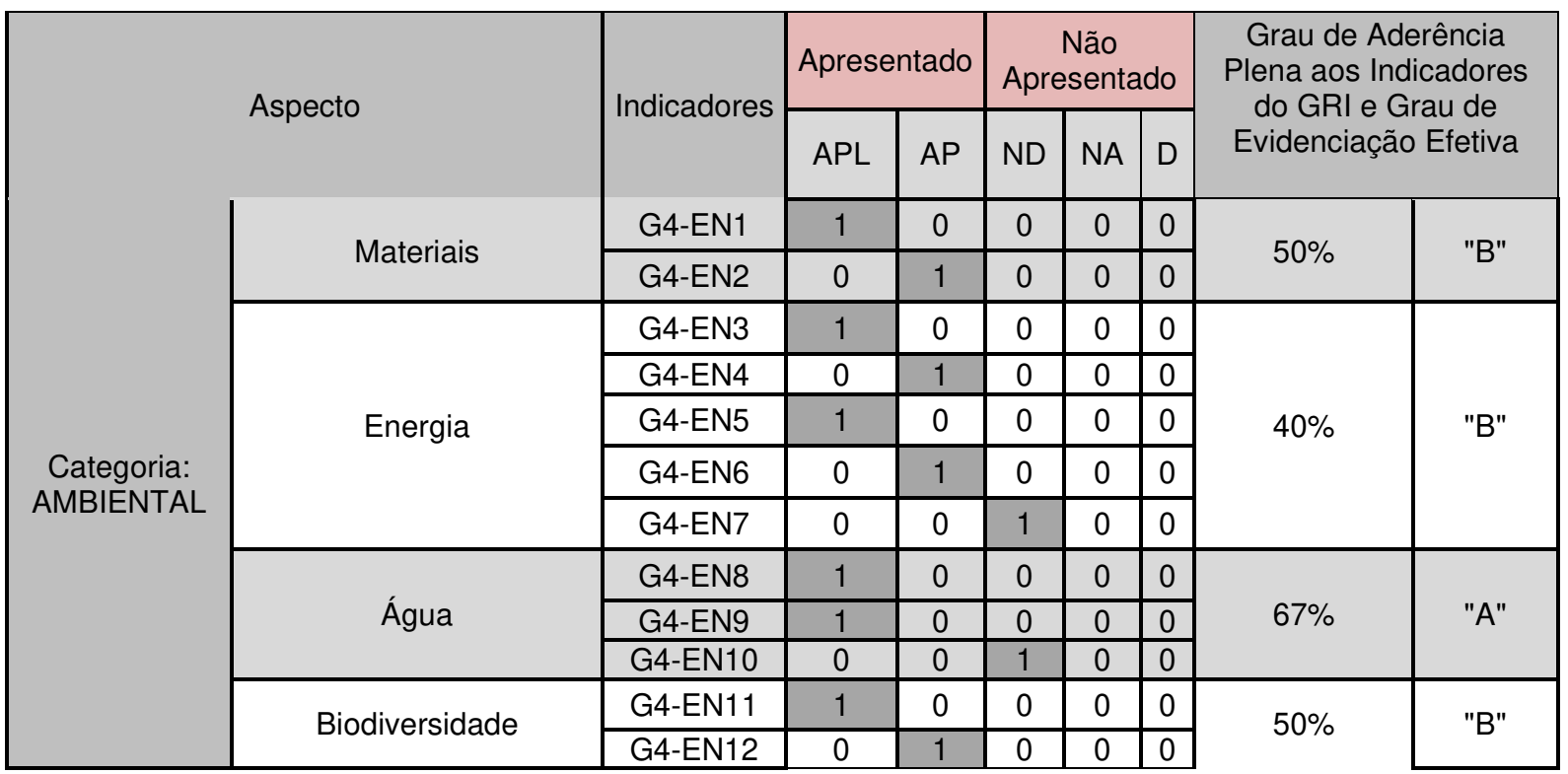




\section{GESTÃO \& SUSTENTABILIDADE}

AMBIENTAL

\section{sunisul}

\begin{tabular}{|c|c|c|c|c|c|c|c|c|}
\hline & G4-EN13 & 1 & 0 & 0 & 0 & 0 & & \\
\hline & G4-EN14 & 0 & 0 & $\overline{0}$ & 0 & 1 & & \\
\hline \multirow{7}{*}{ Emissões } & G4-EN15 & 1 & 0 & 0 & 0 & 0 & \multirow{7}{*}{$71 \%$} & \multirow{7}{*}{ "A" } \\
\hline & G4-EN16 & 1 & 0 & 0 & 0 & 0 & & \\
\hline & G4-EN17 & 1 & 0 & 0 & 0 & 0 & & \\
\hline & G4-EN18 & 1 & 0 & 0 & 0 & 0 & & \\
\hline & G4-EN19 & 1 & 0 & 0 & 0 & 0 & & \\
\hline & G4-EN20 & 0 & 0 & 1 & 0 & 0 & & \\
\hline & G4-EN21 & 0 & 0 & 1 & 0 & 0 & & \\
\hline \multirow{5}{*}{ Efluente e Resíduos } & G4-EN22 & 0 & 0 & 0 & $\overline{1}$ & 0 & \multirow{5}{*}{$80 \%$} & \multirow{5}{*}{ "A" } \\
\hline & G4-EN23 & 1 & 0 & 0 & 0 & 0 & & \\
\hline & G4-EN24 & 1 & 0 & 0 & 0 & 0 & & \\
\hline & G4-EN25 & 1 & 0 & 0 & 0 & 0 & & \\
\hline & G4-EN26 & 1 & 0 & 0 & 0 & 0 & & \\
\hline \multirow{2}{*}{ Produtos e Serviços } & G4-EN27 & 0 & 0 & 1 & 0 & 0 & \multirow{2}{*}{$50 \%$} & \multirow{2}{*}{ "B" } \\
\hline & G4-EN28 & 1 & 0 & 0 & 0 & 0 & & \\
\hline Conformidade & G4-EN29 & 0 & $\overline{0}$ & 0 & 1 & 0 & $0 \%$ & "C" \\
\hline Transporte & G4-EN30 & 0 & 0 & 1 & 0 & 0 & $0 \%$ & "C" \\
\hline Geral & G4-EN31 & 1 & 0 & 0 & 0 & 0 & $100 \%$ & "A" \\
\hline Avaliação Ambiental de & G4-EN32 & 1 & 0 & 0 & 0 & 0 & \multirow{2}{*}{$100 \%$} & \multirow{2}{*}{ "B" } \\
\hline Fornecedores & G4-EN33 & 1 & $\overline{0}$ & 0 & 0 & 0 & & \\
\hline $\begin{array}{l}\text { Mecanismos de Queixas } \\
\text { e Reclamações } \\
\text { Relacionadas a Impactos } \\
\text { Ambientais } \\
\end{array}$ & G4-EN34 & 0 & 0 & 1 & 0 & 0 & $0 \%$ & "C" \\
\hline \multicolumn{2}{|l|}{ Total } & 20 & 4 & 7 & 2 & 1 & $61 \%$ & "B" \\
\hline
\end{tabular}

Fonte: Autores (2019).

Para Travassos et al., (2014), no GAPIE verifica-se o quanto as empresas respeitam as determinações estabelecidas pela GRI. No GEE verifica-se o nível de informação que a empresa está efetivamente oferecendo aos seus usuários em comparação a todo o potencial.

O Quadro 8 sumariza a classificação dos 11 aspectos analisados, em que 06 foram classificados como Alto GAPIE e GEE, são eles: água; emissões; efluente e resíduos; geral e avaliação ambiental de fornecedores, sendo esses dois últimos aspectos determinados com grau máximo de 100\%, o que demonstra um nível de maturidade e responsabilidade da empresa em relação a esses quesitos.

O aspecto "água" é muito relevante, tendo em vista que a empresa é do ramo da fruticultura irrigada, ou seja, sua produção depende profundamente dessa variável, e apesar de este estudo ter classificado como de alto grau de aderência, com o 
percentual de 67\%, considera-se que a empresa deve, urgentemente, implementar estratégias para atingir o grau máximo de $100 \%$, tendo em vista que água é um recurso finito.

O Quadro 8 também mostra que 04 aspectos foram considerados como Médio GAPIE e GEE, a saber: materiais, energia, biodiversidade e produtos e serviços; e 03 foram considerados como Baixo, são eles: Conformidade, Transporte e Mecanismos de Queixas e Reclamações Relacionadas a Impactos Ambientais.

Quadro 8 - Classificação Geral dos Aspectos da empresa analisada

\begin{tabular}{|c|c|c|c|}
\hline Aspecto & \multicolumn{2}{|c|}{ GAPIE e GEE } & Classificação \\
\hline Materiais & $50 \%$ & "B" & Médio \\
\hline Energia & $40 \%$ & "B" & Médio \\
\hline Água & $67 \%$ & "A" & Alto \\
\hline Biodiversidade & $50 \%$ & "B" & Médio \\
\hline Emissões & $71 \%$ & "A" & Alto \\
\hline Efluente e Resíduos & $80 \%$ & "A" & Alto \\
\hline Produtos e Serviços & $50 \%$ & "B" & Médio \\
\hline Conformidade & $0 \%$ & "C" & Baixo \\
\hline Transporte & $0 \%$ & "C" & Baixo \\
\hline Geral & $100 \%$ & "A" & Alto \\
\hline Avaliação Ambiental de Fornecedores & $100 \%$ & "A" & Alto \\
\hline Mecanismos de Queixas e Reclamações & $0 \%$ & "C" & Baixo \\
\hline Relacionadas a Impactos Ambientais & & & \\
\hline Média Total & $61 \%$ & "B" & Médio \\
\hline
\end{tabular}

Fonte: Autores (2019).

O resultado total de todos os indicadores foi na faixa de $61 \%$, nível "B" e classificação de Média Aderência ao GRI.

De forma geral, a empresa apresenta um médio desempenho de classificação. O seu melhor desempenho está nos aspectos geral e avaliação ambiental de Fornecedores; ao passo que os piores desempenhos foram registrados nos aspectos conformidade, transportes e mecanismos de queixas e reclamações relacionadas a impactos ambientais.

Os dados apresentados na Figura 3 permitem a visualização geral dos percentuais de todos os indicadores. Nos valores mais desejáveis de $100 \%$ de aderência (GAPIE e GEE), encontram-se o aspecto geral e de avaliação ambiental de fornecedores, para os anos de 2016 a 2018. 


\section{sunisul}

Figura 3- Gráfico da classificação geral dos aspectos da empresa analisada. A linha azul representa o GAPIE e GEE atingidos por cada aspecto analisado

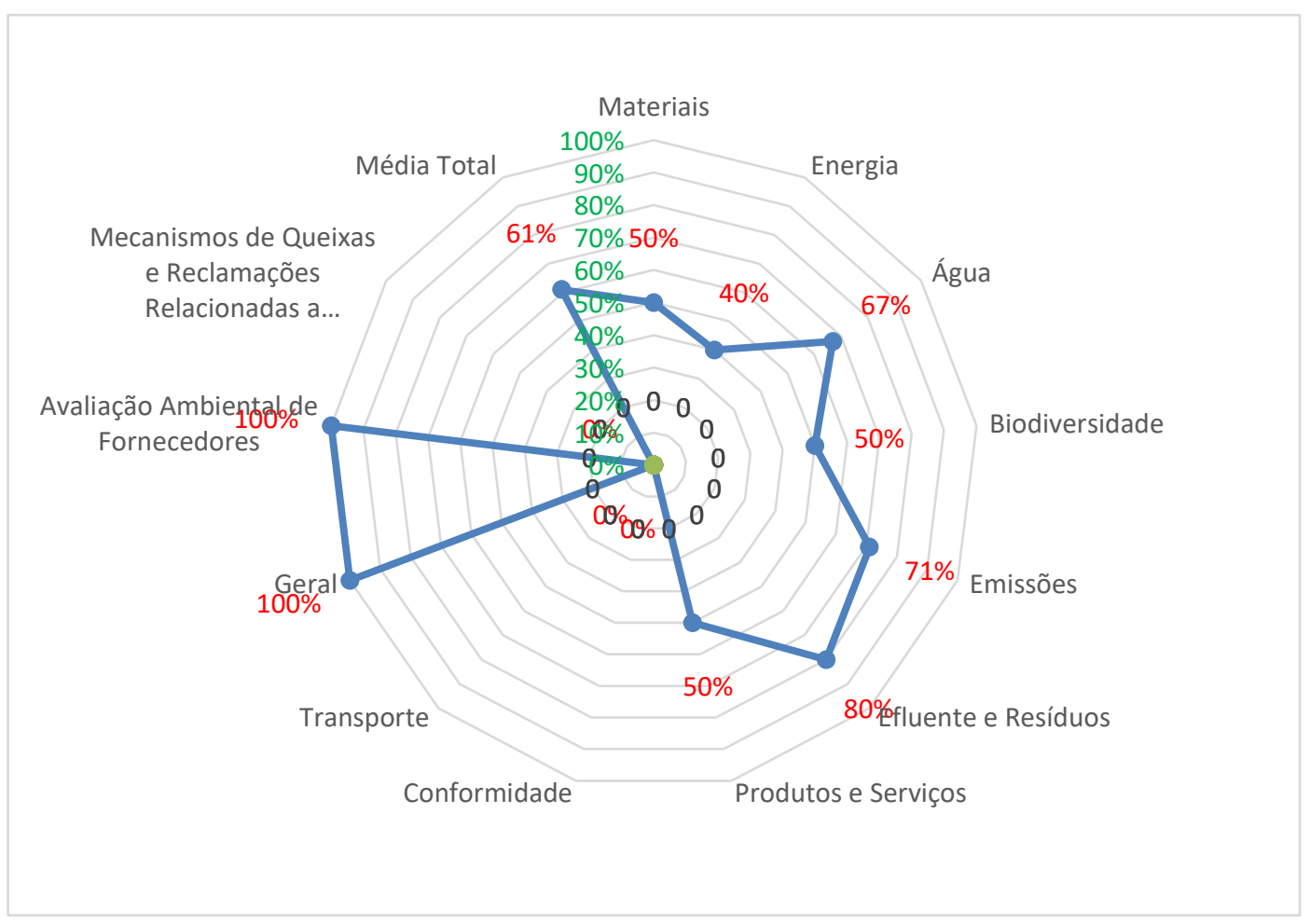

Fonte: Autores (2019).

\section{CONCLUSÃO}

O presente estudo verificou o GAPE e o GEE de uma empresa de Fruticultura Irrigada do Vale do São Francisco em Petrolina - PE nos anos de 2016 a 2018. Para tanto, fez-se necessário contextualizar os conceitos de Gestão Ambiental e o seu papel nas empresas brasileiras; o uso dos Indicadores Ambientais desde a sua origem até a sua importância; e o GRI e o Relatório de Sustentabilidade para descrever seu desempenho frente às questões ambientais.

O resultado apresentado salienta que, mesmo a empresa atuando apenas no segmento agrícola, voltada à produção de fruticultura, possui um nível considerado bom de aderência aos indicadores ambientais.

Quanto à aplicabilidade regional, a pesquisa vem agregar informações, pois, até o momento, não havia nenhuma informação sobre o perfil das empresas do Vale do São Francisco em relação aos parâmetros estudados nesse segmento, o que 


\section{GESTÃO \& SUSTENTABILIDADE AMBIENTAL}

\section{¿unisul}

dificultou as discussões comparativas com outras empresas do mesmo segmento, sendo este estudo um dos primeiros estudos na região.

Esta pesquisa serve como base para futuros estudos sobre esta temática. A divulgação dos indicadores de sustentabilidade das empresas do Vale do São Francisco, Semiárido nordestino, é de peculiar importância a fim de que se tenha uma gestão mais efetiva pelas empresas e para que as políticas públicas caminhem em parceria com o setor empresarial e anseios da sociedade no quesito da sustentabilidade.

\section{REFERÊNCIAS}

ALMEIDA, C. L.; NUNES, A. B. de A. Proposta de indicadores para avaliação de desempenho dos Sistemas de Gestão Ambiental e de Segurança e Saúde no Trabalho de Empresas do ramo de engenharia consultiva. Gest. Prod. Disponível em: http://dx.doi.org/10.1590/0104-530X649.2014. Acesso em: 13 abr. 2019.

ANDRADE, M. M. Introdução à metodologia do trabalho científico. 9. ed. São Paulo: Atlas, 2009.

BRAGA, T. M.; FREITAS, A. P. G.; DUARTE, G. de S.; CAREPA-SOUSA, J. Índices de sustentabilidade municipal: o desafio de mensurar. Nova Economia, Belo Horizonte MG v. 14, n. 3, p.11-33, set./dez. 2004.

CARVALHO, F. M. Análise da utilização dos indicadores essenciais da Global Reporting Initiative nos relatórios sociais em empresas latino-americanas, Dissertação (mestrado) - Universidade Federal do Rio de Janeiro, Rio de Janeiro, RJ, Brasil, 2007.

CARVALHO, F. M.; SIQUEIRA, J.R.M. Análise da utilização dos Indicadores Essenciais da Global Reporting Initiative nos Relatórios Sociais em Empresas LatinoAmericanas. Pensar Contábil, Vol. 9, № 38, 2007.

CASTRO, F. A. R.; SIQUEIRA, J. R. M.; MACEDO, M. A. S. Análise da utilização dos indicadores essenciais da versão "G3", da Global Reporting Initiative, nos relatórios de sustentabilidade das empresas do setor de energia elétrica sul americano. In: SIMPÓSIO DE ADMINISTRAÇÃO DE PRODUÇÃO, LOGÍSTICA E OPERAÇÕES INTERNACIONAIS, 2009, Rio de Janeiro. Anais. Rio de Janeiro, 2009.

CENTRO NACIONAL DE TECNOLOGIAS LIMPAS (CNTL). Manual 7: Indicadores Ambientais e Plano de Monitoramento. Porto Alegre: [s.n.] 2001.

DIAS, L. N. S. Análise da utilização dos indicadores do Global Reporting Initiative nos relatórios sociais em empresas brasileiras, Dissertação (Mestrado) Universidade Federal do Rio de Janeiro, Rio de Janeiro, RJ, Brasil, 2006.

FERNANDES, F. S.; SIQUEIRA, J. R. M.; GOMES, M. Z.. A decomposição do modelo da Global Reporting Initiative (GRI) para avaliação de relatórios de sustentabilidade. Revista do BNDES, v.34, p.101-132, 2010. 


\section{GESTÃO \& SUSTENTABILIDADE AMBIENTAL}

\section{¿unisul}

GLOBAL REPORTING INITIATIVE - GRI. Diretrizes para o Relatório de Sustentabilidade. 2006. Disponível em: <www.globalreporting.org/>. Acesso em 09 maio 2019.

IBGE. PINTEC Sustentabilidade: 40,6\% das empresas inovadoras introduziram inovações com impactos ambientais positivos entre 2015 e 2017. Disponível em https://agenciadenoticias.ibge.gov.br/. Acesso em 15 jul. 2020.

INSTITUTO BRASILEIRO DE RELAÇÕES COM INVESTIDORES (IBRI). Pesquisa IBRI-Deloitte: Qualidade das informações Transparência e integração nas divulgações das companhias abertas. Disponível em: http://www.ibri.org.br/materias/enquete.asp. Acesso em 01 jul. 2020.

MUELLER, A. A. A utilização dos Indicadores de Responsabilidade Social Corporativa e Sua Relação com os Stakeholders - Dissertação apresentada ao Programa de Pós-Graduação em Engenharia de Produção da Universidade Federal de Santa Catarina, Florianópolis SC, 2003.

NETO, M. J; KRÜGER, C. M.; DZIEDZIC, M. Análise de indicadores ambientais no reservatório do Passaúna. Eng. Sanit. Ambient, v. 14, n. 2, p. 205-214, abr./jun. 2009.

NUNES-MAIA, M. F. (2009, jan./abr.). A gestão de resíduos urbanos e suas limitações. Revista Baiana de Tecnologia, Salvador BA, v.7, n.1, p.1120-129, jan./abr. 2009.

OLIVEIRA, C. de M. A noção de diálogo materializada nos relatórios GRI. Dissertação (mestrado) - Universidade Federal do Rio Grande do Sul, Porto Alegre, RS, Brasil, 2017.

PEREIRA, N. S. P.; PEREIRA, C. A.; MONTEIRO, R. P.; PAIXÃO FILHO, J. M. Relatórios de sustentabilidade: ferramenta de interface no desempenho social, econômico e ambiental das organizações. Revista de Auditoria Governança e Contabilidade - RAGC, v. 3, n.5, p. 55-70, 2015.

PHILIPPI JUNIOR, A.; BRUNA, G. C. Curso de gestão ambiental. In: PHILIPPI JUNIOR, A., ROMÉRO, M. A.; BRUNA, G. C. (Orgs.). Política e gestão ambiental. Barueri: Manole, 2004, cap. 18, p. 657- 711.

POLAZ, C. N. M.; TEIXEIRA, B. A. do N. Indicadores de sustentabilidade para a gestão municipal de resíduos sólidos urbanos: um estudo para São Carlos (SP). Eng. Sanit. Ambient. v. 14, n.3, p.411-420, jul/set. 2009.

RIBAS, J. R.; VICENTE, T. V. dos S.; ALTAF, J. G.; TROCCOLI, I. R. Integração de Ações na Gestão Sustentável. Revista Eletrônica de Administração - REAd, ed. 86, v. 2, p. 31-57. 2017.

$\mathrm{ROCHA}$, T. A. C. Análise do relatório GRI enquanto ferramenta para a mensuração da sustentabilidade empresarial. Dissertação (mestrado) Universidade de São Paulo, Ribeirão Preto, SP, Brasil, 2012.

SANTOS, R. F.; GONÇALVES, M. N. Relatório de Sustentabilidade GRI versão G4: as companhias estão seguindo a estrutura e as premissas? $2^{\circ}$ Encontro Anual de Iniciação Científica - XXIV EAIC, IV. Encontro Anual de Iniciação Científica - IV EAIC, set. 2015. 


\section{sunisul}

SILVA, T. L. G. B.; MAIA, L. C. C; LEAL, E. A. Grau de aderência aos indicadores de desempenho ambiental do GRI em um mercado emergente: Uma análise em empresas com potencial poluidor em dois segmentos. Revista Ambiente Contábil Universidade Federal do Rio Grande do Norte, v. 9, n. 1, p. 21-36, 2017.

SGS. RELATÓRIO DE SUSTENTABILIDADE (GRI). 2019. Disponível em https://www.sgsgroup.com.br/pt-br/news/2017/10/relatorio-de-sustentabilidade-gri.

Acesso em 15 jul. 2020

STROBEL, J. S. Modelo para mensuração da sustentabilidade corporativa através de indicadores. Dissertação (mestrado) - Universidade Federal de Santa Catarina, Florianópolis, SC, Brasil, 2005.

SUCENA, E; MARINHO, M. M. O. Environmental disclosure analysis of sustainability reports the brazilian and international brewing industry based on Global Reporting Initiative - GRI. Gestão \& Produção, 26(3), e3120. https://doi.org/10.1590/0104530X3120. 2019.

TAUHATA, S. GRI cria conselho no Brasil com presença de grandes investidores. 2020. Disponível em https://valorinveste.globo.com/mercados/rendavariavel/empresas/noticia/2020/03/03/gri-cria-conselho-no-brasil-com-presenca-degrandes-investidores.ghtml. Acesso em 10 jul. 2020.

TRAVASSOS, S. K. de M.; COSTA, J. I. de F.; SILVA, W. E. da; ARAÚJO, M. de L. Uso dos indicadores essenciais da GRI nos relatórios das empresas dos setores de petróleo, gás e biocombustível e de utilidade pública no Brasil. Revista de Gestão Ambiental e Sustentabilidade - GeAS, v. 3, n. 2, maio/ago. 2014.

YIN, Robert K. Estudo de caso: planejamento e métodos. 2. ed. Porto Alegre: Bookman. 2001. 